\title{
Landau Theory of Fermi Liquid in a Relativistic Nonlinear $(\sigma, \omega)$ Model at Finite Temperature
}

\author{
Schun T. Uechi' ${ }^{1}$ Hiroshi Uechi ${ }^{2}$ \\ ${ }^{1}$ Department of Physics \& Astronomy, The University of Georgia, Athens, GA, USA \\ ${ }^{2}$ Osaka Gakuin University, Osaka, Japan \\ Email: schunuechi@uga.edu, uechi@ogu.ac.jp
}

Received 21 May 2016; accepted 25 July 2016; published 28 July 2016

Copyright (C) 2016 by authors and OALib.

This work is licensed under the Creative Commons Attribution International License (CC BY).

http://creativecommons.org/licenses/by/4.0/

(c) (i) Open Access

\section{Abstract}

Fermi liquid properties of nuclear matter at finite temperature are studied by employing a relativistic nonlinear $(\sigma, \omega)$ model of quantum hadrodynamics (QHD). The relativistic nonlinear $(\sigma, \omega)$ model is one of the thermodynamically consistent $Q H D$ approximations. The QHD approximations maintain the fundamental requirement of density functional theory (DFT). Hence, the finite temperature nonlinear $(\sigma, \omega)$ mean-field approximation can be self-consistently constructed as a conserving approximation. Fermi liquid properties of nuclear matter, such as incompressibility, symmetry energy, first sound velocity and Landau parameters, are calculated with the nonlinear $(\sigma, \omega)$ mean-field approximation, and contributions of nonlinear interactions and finite temperature effects are discussed. Self-consistent structure to an employed approximation as conserving approximation is essential to examine physical quantities at finite temperature. Finite-temperature effects are not large at high density, however, the Fermi ground state, density of states and Fermi-liquid properties may be varied noticeably with a finite temperature $(T \gtrsim 10 \mathrm{MeV})$ at low densities. Low-density finite-temperature and high-density finite-temperature experiments might exhibit physically different results, which should be investigated to understand nuclear manybody phenomena.

\section{Keywords}

Quantum Hadrodynamics (QHD), DFT in Nuclear Matter, Nonlinear Mean-Field Theory, Landau Parameters at Finite Temperature

Subject Areas: Nuclear Physics, Theoretical Physics 


\section{Introduction}

Quantum hadronic theories of hot and dense nuclear matter have been applied to nuclear-structure properties such as proton-nucleus scattering and nuclear matter [1]-[5], and problems of nuclear-astrophysics: neutron stars, baryon-hyperon phase transition, hadron-quark phase transition and quark-gluon plasma. Equations of state of nuclear and hyperonic matter are fundamental to examine properties of neutron star masses, radii, moments of inertia [6]-[8]. It is expected that the phase-transition temperature from hadronic phase to quark-gluon plasma should be of the order of $T=200 \mathrm{MeV}$, and it is hoped that head-on heavy-ion collision experiments may achieve physical values of defining hadron-quark phase transition [9]-[11]. In order to examine wide variety of hypothetical nuclear and astrophysical phenomena, one will need consistent microscopic many-body calculations based on a relativistic quantum field theory at finite temperature [12] [13].

The dynamics of infinite hadronic matter can be checked against conditions of macroscopic conservation laws, such as, the virial theorem [14], the first law of thermodynamics, Gibbs' relation and the corresponding differential laws which are written covariantly [15] [16]. Macroscopic properties are expressed with particle density, energy density, pressure and entropy constructed from basic interactions of particles. Covariant formulations of thermodynamics and thermodynamic consistency in microscopic calculations are explicitly shown in a relativistic formalism. The consistency of microscopic calculations and validity of approximations can be examined through macroscopic conservation laws [17]-[23].

Nuclear many-body theory states that even in the strong interaction region, the effect of the nuclear medium on a specific nucleon about the Fermi energy range can be described by a single particle energy that will be determined by microscopic interactions of particles. For all processes with energy near the Fermi energy, the nucleus may be considered as a gas of (quasi-) particles [4] [24] [25], which satisfies macroscopic conservation laws, and we have a picture that self-consistently determined or dressed single particles move within the mean-field potential of nucleons. The dynamically determined single particle energy constructed from a basic Hamiltonian or a Lagrangean and the self-consistent single quasiparticle energy of nuclear matter must be equivalent at the Fermi surface, which is known as Landau's requirement of quasiparticles [26]-[28] or the requirement of density functional theory (DFT) [29] [30].

We employ a relativistic nonlinear $(\sigma, \omega)$ effective model of hadrons [8] [31], extended from a relativistic quantum field theory, Quantum Hadrodynamics (QHD) [32] [33], and the nonlinear mean-field approximation is applied to nuclear matter in order to investigate Fermi liquid properties of nuclear matter at finite temperature.

The mean-field approximation (Walecka model) to the theory of QHD produces a thermodynamically consistent field theoretical approximation. One can directly show that dynamical single particle energy defined in Green's function and quasiparticle energy defined by Landau's fundamental requirement are equal, and consequently, Fermi liquid properties of nuclear matter are discussed consistently at zero temperature [34]-[36]. The self-consistency, equality of dynamical and quasiparticle single particle energies, can be proved only if nonlinear interactions are properly renormalized. The fundamental requirement of conserving approximation or DFT is satisfied. The nonlinear $(\sigma, \omega)$ effective model is a conserving approximation [37] [38], which is also essential for self-consistent finite temperature approximations. Nonlinear interactions should be properly renormalized as effective masses and effective coupling constants to be a conserving approximation.

The high-density, high-energy phenomena would require a relativistic and non-equilibrium kinetic theory at finite temperature. The relation between quasiparticle scattering amplitudes and Landau parameters is necessary for reasonable approximations and calculations for finite temperature properties of Fermi liquids [39]. Relativistic hadronic models are also essential to examine nuclear fissions and cluster radioactivities in terms of conservation laws and self-consistency [40]-[42]. Astrophysical problems such as the formation of neutron stars require finite temperature and nonlinear hadronic approximations [15] [16] [33]; density and temperature inside stars will increase toward the center of a star, which is expected to produce pion condensations, hyperon generations and hadron-quark neutron stars [7] [8] [10] [31].

It is important to know finite temperature effects on many-body systems of quasiparticles. Based on self-consistency and DFT, we extend the nonlinear $(\sigma, \omega)$ effective model to the finite temperature mean-field approximation and examine Landau parameters, finite temperature effects on Fermi liquid properties of nuclear matter.

\section{Macroscopic Properties at Zero Temperature}

The distribution function, $n(k)$, is a function of quasiparticle momentum $\boldsymbol{k}$ and single particle energy $E(\boldsymbol{k})$ 
$\left(0 \leq k \leq k_{F}\right)$ directly connected to the ground state energy of Fermi particles at $T=0$, and the single particle energy is equal to the chemical potential, $\mu_{0}$. The distribution function, $n(k)$, has a characteristic property at $T$ $=0: 1\left(k \leq \mu_{0}\right)$ and $0\left(k>\mu_{0}\right)$, and physical quantities of the ground state of nuclear matter are described by the Fermi momentum, $k_{F}$, or the baryon density, $\rho_{B}=\zeta k_{F}^{3} / 6 \pi^{2}$ where $\zeta$ is a spin-isospin degeneracy number $(\zeta=$ 2 for neutron matter and $\zeta=4$ for nuclear matter).

The current nonlinear $(\sigma, \omega)$ mean-field lagrangian is defined by:

$$
\mathcal{L}=\bar{\psi}\left[\gamma_{\mu}\left(i \partial^{\mu}-g_{\omega} \omega^{\mu}\right)-\left(M-g_{\sigma} \phi\right)\right] \psi-\frac{1}{2} m_{\sigma}^{2} \phi^{2}-\frac{g_{\sigma 3}}{3 !} \phi^{3}-\frac{g_{\sigma 4}}{4 !} \phi^{4}+\frac{1}{2} m_{\omega}^{2} \omega_{\mu}^{2}+\frac{g_{\omega 4}}{4 !}\left(\omega_{\mu} \omega^{\mu}\right)^{2},
$$

and meson quantum fields are replaced with classical fields: $\hat{\phi} \rightarrow \phi, \hat{\omega}_{\mu}=(\hat{\omega}, \hat{\boldsymbol{\omega}}) \rightarrow\left(\omega_{0}, \boldsymbol{\omega}\right)$, (all Greek suffixes run as $\mu=0,1,2,3)$. The replacement of meson quantum fields by classical fields in mean-field lagrangians generally produces Hartree approximation [22] [23] [32]. Generalized nonlinear $(\sigma, \omega, \rho)$ mean-field approximations are discussed in [6] [33] and chap. 6 in [8].

The hadron masses are chosen as $M=939, m_{\sigma}=550, m_{\omega}=783 \mathrm{MeV}$. The coupling constants are fixed so as to produce the nuclear matter saturation property: $\mathcal{E} / \rho_{B}-M=-15.75 \mathrm{MeV}$ at $k_{F}=1.30 \mathrm{fm}^{-1}$. The saturation condition leads to the coupling constants: $g_{\sigma}=9.298, g_{\omega}=10.660, g_{\sigma 3}=200.0(\mathrm{MeV}), g_{\sigma 4}=350.0$, and $g_{\omega 4}=$ 350.0. These coupling constants yield, incompressibility $K=333.4 \mathrm{MeV}$ and symmetry energy, $a_{4}=15.3 \mathrm{MeV}$. The maximum mass of neutron stars produced by the nonlinear model is $M_{\text {star }} / M_{\odot}=1.98\left(M_{\odot}\right.$ is the solar mass). In the following calculations, natural units are used: $\hbar=c=k_{B}=1$.

The equations of motion for the scalar and vector mesons are given by

$$
\begin{aligned}
& m_{\sigma}^{2} \phi+\frac{g_{\sigma 3}}{2 !} \phi^{2}+\frac{g_{\sigma 4}}{3 !} \phi^{3}=g_{\sigma} \rho_{\sigma}, \\
& m_{\omega}^{2} \omega_{\mu}+\frac{g_{\omega 4}}{3 !}\left(\omega_{\nu} \omega^{\nu}\right) \omega_{\mu}=g_{\omega} \rho_{B \mu},
\end{aligned}
$$

where $\rho_{\sigma} \equiv\langle\bar{\psi} \psi\rangle$ is the scalar source; $\rho_{B \mu}=\left\langle\bar{\psi} \gamma_{\mu} \psi\right\rangle=\left(\rho_{B}, \boldsymbol{j}_{B}\right)$ is the baryon density and baryon current density. The explicit expression of scalar source $\rho_{\sigma}$ is derived from minimization of energy density with respect to $\phi$, or self-consistent condition of $\delta \mathcal{E} / \delta n_{i}=E\left(k_{i}\right)$ :

$$
\rho_{\sigma}=\sum_{i} n_{i} \frac{M^{*}}{E^{*}\left(k_{i}\right)}
$$

where $E^{*}(k)=\left(k^{2}+M^{* 2}\right)^{1 / 2}$, and the nucleon effective mass is defined by $M^{*}=M-g_{\sigma} \phi$. The baryon density, $\rho_{B}$, is given by

$$
\rho_{B}=\sum_{i} n_{\kappa_{i}}=\frac{\zeta}{6 \pi^{2}} k_{F}^{3}
$$

where $\zeta$ is a degeneracy factor: $\zeta=4$ for nuclear matter and $\zeta=2$ for neutron matter. The baryon current, $\boldsymbol{j}_{B}$, is similarly obtained from self-consistent condition as,

$$
\dot{j}_{B}=\sum_{i} n_{\kappa_{i}} \frac{\boldsymbol{\kappa}_{i}}{E^{*}\left(\boldsymbol{\kappa}_{i}\right)}
$$

where the momentum $\boldsymbol{\kappa}$ is defined by [34],

$$
\boldsymbol{\kappa}=k-g_{\omega} \omega .
$$

The single particle energy is given by Green's function formalism, and the Fermi energy, $E\left(\boldsymbol{k}_{F}\right)$, and chemical potential defined by the Gibbs' relation in thermodynamics can be proved to be equal in the mean-field approximations to QHD, which shows that the requirement of conserving approximations [17] [18] [22] [23] and DFT [29] [30] is maintained. The requirement of self-consistency is not automatically satisfied in approximations and must be checked in all self-consistent approximations. Even if some calculations compared to data seem to be reasonable, approximations which do not maintain the requirement of conserving approximations cannot be accepted.

The Green's function is defined by Schwinger-Dyson equation, and the baryon Green's function with renor- 
malized dynamical variables has a similar structure as the noninteracting Green's function, which assumes the existence of the (on-shell) single quasiparticle energy, $E(k)$. The Green's function $G(k)$ is decomposed as Dirac and Feynman terms: $G(k)=G_{D}(k)+G_{F}(k)$ [32]. The Dirac term, $G_{D}(k)$, expresses the propagation of positive energy Fermi-sea particles at finite baryon density, and $G_{F}(k)$ is the interacting Feynman propagator. As discussed considerably in [21]-[23] [32], we will employ the renormalized Fermi-sea particle approach to extract finite and physically meaningful contributions by assuming the Green function as $G_{D}(k)$. Therefore, it is essential that physical quantities such as the single particle energy, effective masses of baryons and mesons and effective coupling constants must be self-consistently renormalized by the fundamental requirement of the density functional theory.

The energy density and pressure are calculated by way of energy-momentum tensor and Green's function as [6] [8] [32],

$$
\begin{aligned}
& \mathcal{E}=\frac{\zeta}{2 \pi^{2}} \int_{0}^{k_{F}} \mathrm{~d} k k^{2} E(k)+\frac{1}{2} m_{\sigma}^{2} \phi^{2}+\frac{g_{\sigma 3}}{3 !} \phi^{3}+\frac{g_{\sigma 4}}{4 !} \phi^{4}-\frac{1}{2} m_{\omega}^{2} \omega_{\mu}^{2}-\frac{g_{\omega 4}}{4 !}\left(\omega_{\mu} \omega^{\mu}\right)^{2}, \\
& p=\frac{\zeta}{3 \pi^{2}} \int_{0}^{k_{F}} \mathrm{~d} k \frac{k^{4}}{E^{*}(k)}-\frac{1}{2} m_{\sigma}^{2} \phi^{2}-\frac{g_{\sigma 3}}{3 !} \phi^{3}-\frac{g_{\sigma 4}}{4 !} \phi^{4}+\frac{1}{2} m_{\omega}^{2} \omega_{\mu}^{2}+\frac{g_{\omega 4}}{4 !}\left(\omega_{\mu} \omega^{\mu}\right)^{2} .
\end{aligned}
$$

The matrix structure of the baryon self-energy will be reduced generally to the following form:

$$
\Sigma(k)=\Sigma^{s}(k)-\gamma_{\mu} \Sigma^{\mu}(k)=\Sigma^{s}\left(|\boldsymbol{k}|, k^{0}\right)-\gamma^{0} \Sigma^{0}\left(|\boldsymbol{k}|, k^{0}\right)+\gamma \cdot \boldsymbol{k} \Sigma^{i}\left(|\boldsymbol{k}|, k^{0}\right)
$$

and so, we have three independent self-energy functions, $\Sigma^{s}(k), \Sigma^{0}(k)$ and $\Sigma^{i}(k)=\boldsymbol{k} \Sigma^{v}(k) \quad(i=x, y, z$, the space-homogeneity is assumed). The self-consistent dynamical variables (all dynamical variables are functions of $|\boldsymbol{k}|, k^{0}$ and $k_{F}$ ) are renormalized as:

$$
\begin{aligned}
& M^{*}(k) \equiv M+\Sigma^{s}(k), \quad \boldsymbol{k}^{*}(k) \equiv \boldsymbol{k}\left(1+\Sigma_{\omega}^{v}(k)\right), \\
& k^{* 0} \equiv E^{*}(k) \equiv\left(\boldsymbol{k}^{* 2}(k)+M^{*}(k)^{2}\right)^{1 / 2}, \\
& k^{* \mu} \equiv k^{\mu}+\Sigma_{\omega}^{\mu}(k)=\left(k^{0}+\Sigma_{\omega}^{0}(k), \boldsymbol{k}^{*}(k)\right), \quad k^{0}=E(k) .
\end{aligned}
$$

The self-consistent effective masses of hadrons should be determined from equations of motion, Green's functions for baryons and mesons and the condition of self-consistency, $\delta \mathcal{E} / \delta n(k)$. They are given by [22] [23]:

$$
\begin{aligned}
& M^{*}=M-g \phi \\
& m_{\sigma}^{* 2}=m_{\sigma}^{2}+\frac{g_{\sigma 3}}{2 !} \phi+\frac{g_{\sigma 4}}{3 !} \phi^{2} \\
& m_{\omega}^{* 2}=m_{\omega}^{2}+\frac{g_{\omega 4}}{3 !} \omega_{\mu} \omega^{\mu} .
\end{aligned}
$$

Scalar and vector self-energies are related to fields and sources by:

$$
\begin{aligned}
& \Sigma^{s}=-g_{\sigma} \phi=-\frac{g_{\sigma}^{2}}{m_{\sigma}^{* 2}} \rho_{\sigma}, \\
& \Sigma_{\omega}^{0}=-g_{\omega} \omega_{0}=-\frac{g_{\omega}^{2}}{m_{\omega}^{* 2}} \rho_{B} \text { and } \Sigma_{\omega}^{i}=-g_{\omega} \omega^{i}=-\frac{g_{\omega}^{2}}{m_{\omega}^{* 2}} j_{B}^{i} \quad(i=x, y, z) .
\end{aligned}
$$

One should note that the effective masses (10) and self-energies (11) are derived from self-consistent condition, $\delta \mathcal{E} / \delta \rho_{B}=E\left(k_{F}\right)$, and equations of motion for mesons. Hence, renormalized effective masses and self-energies are consistent with requirements of thermodynamic consistency and the density functional theory.

The saturation curves of binding energy at $T=0$ are given in Figure 1, in order to show that the linear $(\sigma, \omega)$ and nonlinear $(\sigma, \omega)$ mean-field approximations in QHD model maintain the fundamental requirement of nuclear matter, which is taken in the current calculation as, $\rho_{B}=0.148 \mathrm{fm}^{-3}, \mathcal{E} / \rho_{B}-M=-15.75 \mathrm{MeV}$ [19]-[21] [32]. In Figure 1, the smooth curve at saturation (NHA) produces a small incompressibility, $K_{\mathrm{NHA}}=333.0 \mathrm{MeV}$, compared to $K_{\mathrm{LHA}}=530.3 \mathrm{MeV}$ of LHA binding energy curve, generating a stiff equation of state. A mean-field 


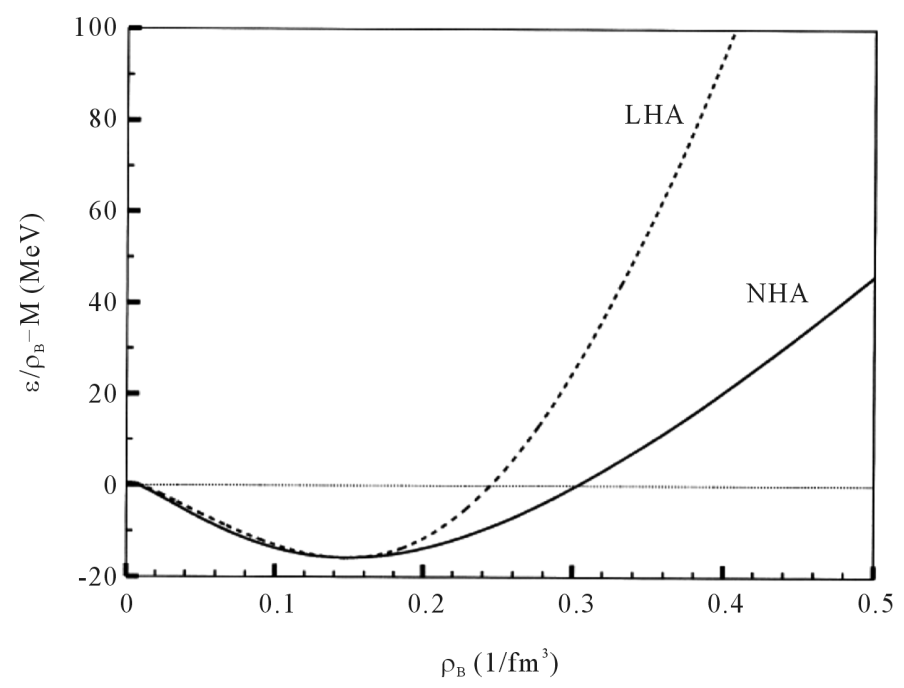

Figure 1. Binding energies of symmetric nuclear matter at $T=0$. The dotted-line is for LHA (linear $\sigma, \omega$ Hartree approximation), and solid-line is for NHA (nonlinear $\sigma$, $\omega$ Hartree approximation), which maintain the saturation condition: $\rho_{B}=0.148 \mathrm{fm}^{-3}$, $\mathcal{E} / \rho_{B}-M=-15.75 \mathrm{MeV}$. The saturation curve of NHA is produced by the coupling constants, $g_{\sigma}=9.298, g_{\omega}=10.660, g_{\sigma 3}=200.0(\mathrm{MeV}), g_{\sigma 4}=350.0$, and $g_{\omega 4}=350.0$, resulting in incompressibility $K=333.4 \mathrm{MeV}$ and symmetry energy, $a_{4}=15.3 \mathrm{MeV}$.

$(\sigma, \omega)$ calculation usually produces a small value of symmetry energy, but it is improved by including $\rho$-meson, or extending to Hartree-Fock, Bruckner HF approximations [21] [32] [43]-[46].

Effective masses of hadrons are shown in Figure 2. The effective mass of nucleon, $M^{*} / M \sim 0.72$ at saturation density, decreases when the baryon density $\rho_{B}$ increases, whereas effective masses of mesons slowly increase $\left(m_{\sigma}^{*} / m_{\sigma} \sim 1.08\right.$ and $m_{\omega}^{*} / m_{\omega} \sim 1.02$ at saturation density).

\section{Macroscopic Properties at Finite Temperature}

To describe the system at finite temperature, we need a thermodynamic potential and partition function that will select the correct ground state in the limit $T \rightarrow 0$ [15] [16]. In the current calculations, the nonlinear $(\sigma, \omega)$ mean-field is reproduced at $T \rightarrow 0$. Thus, we are naturally directed to define

$$
Z=\operatorname{Tr} \exp [-(\hat{H}-\mu \hat{B}-\boldsymbol{v} \cdot \hat{\boldsymbol{P}}) / T] \equiv \exp [-\Omega(T, V, \mu, \boldsymbol{v})],
$$

and the thermodynamic potential in our nonlinear $(\sigma, \omega)$ model is

$$
\begin{aligned}
\Omega(T, V, \mu, v)= & -T \ln Z \\
= & -\frac{1}{2} m_{\sigma}^{2} \phi^{2}-\frac{g_{\sigma 3}}{3 !} \phi^{3}-\frac{g_{\sigma 4}}{4 !} \phi^{4}+\frac{1}{2} m_{\omega}^{2} \omega_{\mu}^{2}+\frac{g_{\omega 4}}{4 !}\left(\omega_{\mu} \omega^{\mu}\right)^{2} \\
& -T \sum_{i}\left[\ln \left(1+\mathrm{e}^{-\left(E^{*}(\kappa)-v \cdot \kappa-v\right) / T}\right)+\ln \left(1+\mathrm{e}^{-\left(E^{*}(\kappa)+v \times \kappa+v\right) / T}\right)\right],
\end{aligned}
$$

where $\boldsymbol{v}$ is a fluid velocity and $\hat{\boldsymbol{P}}$ is a momentum density in nuclear matter.

In finite temperature nuclear matter, particle anti-particle distribution functions are given by,

$$
\begin{aligned}
& n_{\kappa_{i}} \equiv n_{T}(\boldsymbol{\kappa}, v, \boldsymbol{v})=\left(1+\mathrm{e}^{\left(E^{*}(\boldsymbol{\kappa})-\boldsymbol{v} \cdot \boldsymbol{\kappa}-v\right) / T}\right)^{-1}, \\
& \bar{n}_{\kappa_{i}} \equiv \bar{n}_{T}(\boldsymbol{\kappa}, \nu, \boldsymbol{v})=\left(1+\mathrm{e}^{\left(E^{*}(\boldsymbol{\kappa})+v \cdot \boldsymbol{\kappa}+v\right) / T}\right)^{-1},
\end{aligned}
$$

where the single particle energy is: $E(\boldsymbol{\kappa})=E^{*}(\boldsymbol{\kappa})-\Sigma_{\omega}^{0}=\left(\boldsymbol{\kappa}^{2}+M^{* 2}\right)^{1 / 2}-\Sigma_{\omega}^{0}$. The momentum $\boldsymbol{\kappa}$ is given by 


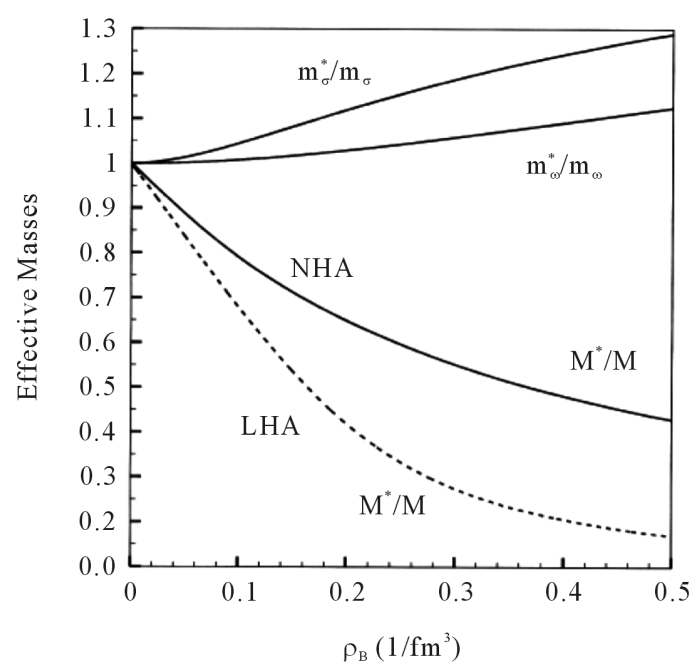

Figure 2. The effctive masses of nucleons and mesons in NHA (solid-lines) and LHA (dotted-line). The meson masses in LHA are constant: $m_{\sigma}$ and $m_{\omega}$. The effective masses of mesons are produced by nonlinear interactions: $m_{\sigma}^{*}$ and $m_{\omega}^{*}$.

$$
\boldsymbol{\kappa}=\boldsymbol{k}-g_{\omega} \boldsymbol{\omega}=\boldsymbol{k}-\frac{g_{\omega}^{2}}{m_{\omega}^{* 2}} \boldsymbol{j}_{B},
$$

where $\boldsymbol{j}_{B}$ is the baryon current:

$$
\dot{j}_{B}=\sum_{i} \frac{\boldsymbol{\kappa}_{i}}{E^{*}\left(\boldsymbol{\kappa}_{i}\right)}\left(n_{\kappa_{i}}+\bar{n}_{\kappa_{i}}\right) .
$$

The self-consistency of nonlinear $(\sigma, \omega)$ approximation requires

$$
v=\mu_{T}-\frac{g_{\omega}^{2}}{m_{\omega}^{* 2}}\left(\rho_{B}-\boldsymbol{v} \cdot \dot{j}_{B}\right) .
$$

The chemical potential, $\mu_{T}$, is equal to the single particle energy at finite temperature $E\left(k_{F}\right)$, and the baryon density, $\rho_{B}(T)$, is given by

$$
\rho_{B}(T)=\sum_{i}\left(n_{\kappa_{i}}-\bar{n}_{\kappa_{i}}\right),
$$

which is denoted as $\rho_{B}$ for simplicity at finite temperature computations.

The equation of motion for scalar meson, which leads to the correct ground state in the limit $T \rightarrow 0$ and consistent with thermodynamic potential (13), is given by

$$
m_{\sigma}^{2} \phi+\frac{g_{\sigma 3}}{2 !} \phi^{2}+\frac{g_{\sigma 4}}{3 !} \phi^{3}=g_{\sigma} \sum_{i} \frac{M^{*}}{\left(\boldsymbol{\kappa}^{2}+M^{* 2}\right)^{1 / 2}}\left(n_{\kappa_{i}}+\bar{n}_{\kappa_{i}}\right) .
$$

The scalar field, $\phi$, is related to the nucleon effective mass $M^{*}$ as $\phi=\left(M-M^{*}\right) / g_{\sigma}$, and the equation of motion for vector meson is:

$$
\begin{aligned}
& m_{\omega}^{2} \omega_{0}+\frac{g_{\omega 4}}{3 !}\left(\omega_{\nu} \omega^{v}\right) \omega_{0}=g_{\omega} \rho_{B}, \\
& m_{\omega}^{2} \omega_{i}+\frac{g_{\omega 4}}{3 !}\left(\omega_{\nu} \omega^{v}\right) \omega_{i}=g_{\omega} \boldsymbol{j}_{B}=g_{\omega} \sum_{i} \frac{\boldsymbol{\kappa}_{i}}{E^{*}\left(\boldsymbol{\kappa}_{i}\right)}\left(n_{\kappa_{i}}+\bar{n}_{\kappa_{i}}\right) .
\end{aligned}
$$

The energy density, pressure, entropy density and momentum density in the nonlinear $(\sigma, \omega)$ model are directly 
derived from the thermodynamic potential (13):

$$
\begin{gathered}
\mathcal{E}=\frac{1}{2} m_{\sigma}^{2} \phi^{2}+\frac{g_{\sigma 3}}{3 !} \phi^{3}+\frac{g_{\sigma 4}}{4 !} \phi^{4}-\frac{1}{2} m_{\omega}^{2} \omega_{\mu}^{2}-\frac{g_{\omega 4}}{4 !}\left(\omega_{\mu} \omega^{\mu}\right)^{2}+g_{\omega} \omega_{0} \rho_{B}+\sum_{i} E^{*}\left(\kappa_{i}\right)\left(n_{\kappa_{i}}+\bar{n}_{\kappa_{i}}\right), \\
p=-\frac{1}{2} m_{\sigma}^{2} \phi^{2}-\frac{g_{\sigma 3}}{3 !} \phi^{3}-\frac{g_{\sigma 4}}{4 !} \phi^{4}+\frac{1}{2} m_{\omega}^{2} \omega_{\mu}^{2}+\frac{g_{\omega 4}}{4 !}\left(\omega_{\mu} \omega^{\mu}\right)^{2}-T \sum_{i}\left[\ln \left(1-n_{\kappa_{i}}\right)+\ln \left(1-\bar{n}_{\kappa_{i}}\right)\right] \\
s=-\sum_{i}\left[n_{\kappa_{i}} \ln n_{\kappa_{i}}+\left(1-n_{\kappa_{i}}\right) \ln \left(1-n_{\kappa_{i}}\right)+\bar{n}_{\kappa_{i}} \ln \bar{n}_{\kappa_{i}}+\left(1-\bar{n}_{\kappa_{i}}\right) \ln \left(1-\bar{n}_{\kappa_{i}}\right)\right], \\
\mathcal{P}=\frac{g_{\omega}^{2}}{m_{\omega}^{* 2}} \rho_{B} \boldsymbol{j}_{B}+\sum_{i} \kappa_{i}\left(n_{\kappa_{i}}-\bar{n}_{\kappa_{i}}\right) .
\end{gathered}
$$

Note that the coupling constants are fixed so as to produce the nuclear matter saturation property: $\mathcal{E} / \rho_{B}-M=$ $-15.75 \mathrm{MeV}$ at $k_{F}=1.30 \mathrm{fm}^{-1}$ at $T=0$, resulting in incompressibility $K=333.4 \mathrm{MeV}$ and symmetry energy, $a_{4}=$ $15.3 \mathrm{MeV}$.

Though nonlinear coupling constants are introduced as free parameters to the model, the saturation conditions, self-consistency and density-dependent nonlinear interactions restrict the values of nonlinear coupling constants [6] [8] [10]. The restrictions to coupling constants become strict when spontaneous symmetry breaking mechanism is used to produce the chiral $(\sigma, \pi, \omega)$ mean-field model [8] [31].

It is necessary to check whether or not generated physical quantities satisfy macroscopic conservation laws. Thermodynamic relations are discussed in the Ref. [15] [16], and one can directly check the following fundamental thermodynamic relation, Gibbs' relation, from the above equations,

$$
\mathcal{E}+p-T s=\mu_{T} \rho_{B}+\boldsymbol{v} \cdot \mathcal{P},
$$

and in the rest frame of nuclear matter, or a comoving frame defined by $\boldsymbol{v}=0$, it becomes $\mathcal{E}+p-T \sigma=\mu_{T} \rho_{B}$. The thermodynamic functions are self-consistently solved by choosing constants, $T, v$ and $v$, and solving Equations (20) and (21) by iteration for the baryon and meson effective masses, baryon density and current.

The concept of Fermi surface at finite temperature is introduced by the relation:

$$
E\left(\boldsymbol{k}_{F}\right)=\mu_{T} \text {. }
$$

Equation (27) self-consistently determines the finite temperature Fermi-momentum $k_{F}(t)$. It is assumed at finite temperature that there exists a chemical potential such that for $E(\boldsymbol{k})$ appreciably greater and smaller than $\mu_{T}$, the particle distribution function behaves as:

$$
n_{T}(\boldsymbol{k}, E(\boldsymbol{k}))= \begin{cases}0 & \text { for } E(\boldsymbol{k})>\mu_{T}, \\ 1 & \text { for } E(\boldsymbol{k}) \leq \mu_{T},\end{cases}
$$

in the rest frame of nuclear matter with the limit of the baryon current, $\boldsymbol{j}_{B} \rightarrow 0$. This allows us to change the variable $\boldsymbol{\kappa}$ to $\boldsymbol{k}$, and the Fermi surface at finite temperature $T$ is defined by

$$
E\left(k_{F}(T)\right)=\mu_{T}=v-\Sigma_{\omega}^{0},
$$

where $v$ is the chemical potential at $T=0$.

At a finite temperature and a low density, it is known that the sharp Fermi surface will be smoothed out, which is also perceived by the disappearance of solutions to Equation (27) at low densities. The stable ground state of Fermi-liquid at $T=0$ is defined by a sharp Fermi energy defined by energy density, and the quasi-particle energy and Pauli exclusion principle are expressed simultaneously. In a finite temperature system, a sharp Fermi surface is smeared out by the effect of temperature agitation. Especially, Fermi energy at low density is completely smeared out and used, for example, to effective masses of hadrons and single particle energy, resulting in the increase of effective masses and binding energies shown in Figure 3 and Figure 4. These characteristics are consistent with those discussed in nonrelativistic calculations of ${ }^{3} \mathrm{He}$ [27].

The definition of Fermi surface is given by the condition, $E^{*}\left(k_{F}\right)=v$, or (29), and hence, solutions do not exist when $M^{*}>v$. These phenomena appear at low densities in a finite temperature (see Figure 3). It indicates that Fermi energy is absorbed to $M^{*}$ or single particle energy, $E(k)$, resulting in the disappearance of 


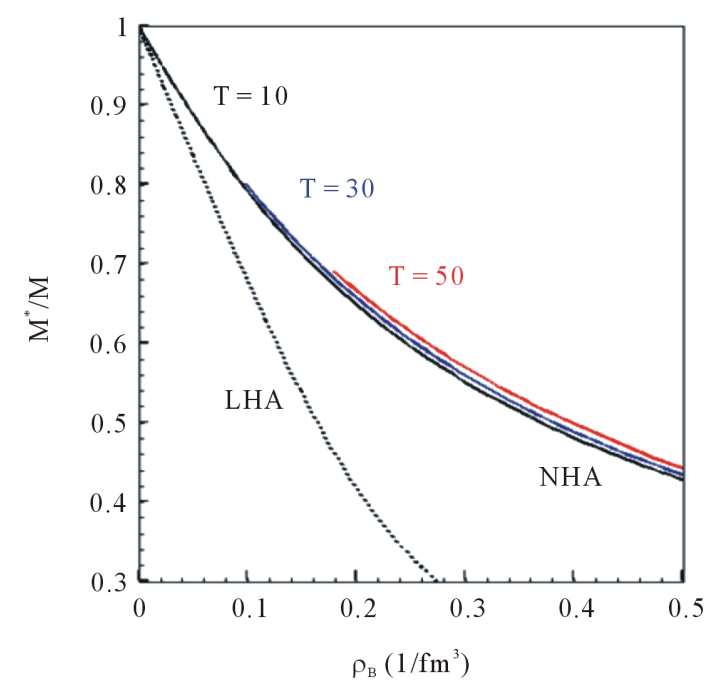

Figure 3. The effective masses of nucleons at finite temperatures: $T=$ $0, T=10, T=30, T=50 \mathrm{MeV}$. The results of $T=0$ and $T=10$ are similar.

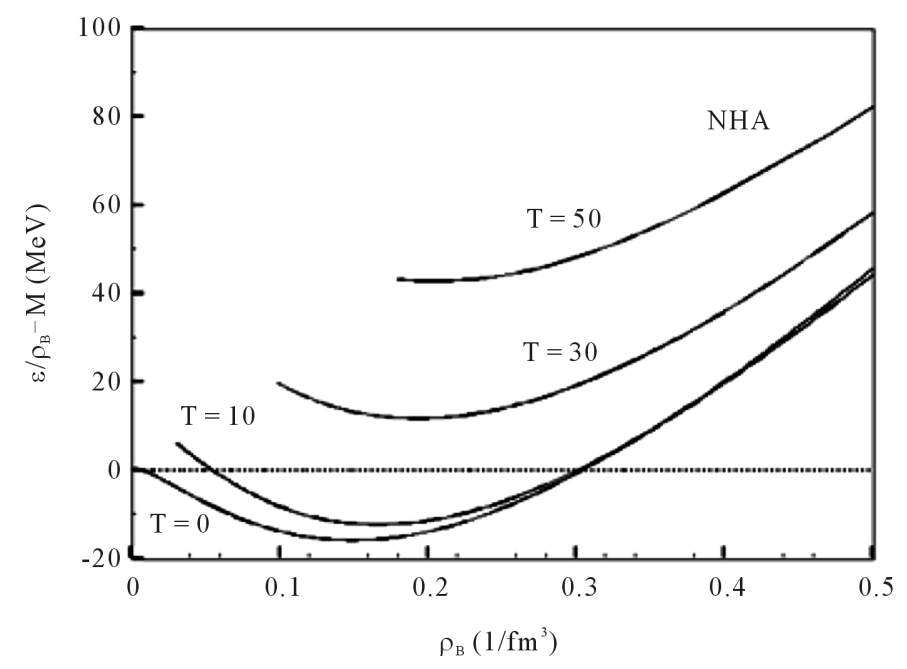

Figure 4. The temperature effect on binding energy of symmetric nuclear matter. Solid-lines are solutions to Fermi-liquid sphere defined by (29). The onset of Fermi-liquid sphere is gradually shifting to a higher density with increasing temperature.

Fermi surfaces (the effective masses cannot be directly calculated because Fermi surface does not exist in the low densities: $\left.E^{*}\left(k_{F}\right)>v\right)$. The Fermi surface exists in the densities, $\rho_{B} \geq 0.030\left(1 / \mathrm{fm}^{3}\right), M^{*} / M \leq 0.93$ at $T=10 \mathrm{MeV} ; \quad \rho_{B} \geq 0.098\left(1 / \mathrm{fm}^{3}\right), M^{*} / M \leq 0.80$ at $T=30 \mathrm{MeV}$ and $\rho_{B} \geq 0.179\left(1 / \mathrm{fm}^{3}\right), M^{*} / M \leq 0.69$ at $T=50 \mathrm{MeV}$.

When baryon density is low, the Fermi surface is gradually smeared out and vanishes as temperature increases. With a fixed finite temperature, the Fermi energy (27) gradually becomes sharp as baryon density increases. The effect of temperature appears at low densities when the thermal energy exceeds Fermi energy and unfreezes the ground state energy of nuclear matter, indicating a gas-liquid phase transition of nuclear matter. The Fermi liquid analysis is confined in higher densities shown in nucleon effective masses and binding energies at $T \neq 0$ in Figure 3 and Figure 4. Hadron effective masses are thermally increased slightly at low densities by absorbing Fermi ground state energy, while the quasiparticle energy and Fermi ground state (Fermi surface) are supposed at all densities in other calculations [4] [24]-[28]. 
When the finite temperature Fermi-momentum $k_{F}(T)$ and zero-temperature Fermi-momentum $k_{F}$ are compared at the same baryon density $\rho_{B}$, one can check $k_{F}(T)$ in (29) is smaller than $k_{F}$ at low densities, and $k_{F}(T) \approx k_{F}$ at high densities. The Fermi-momentum $k_{F}(T)$ is the radius of a smeared Fermi surface and it is conveniently used for numerical calculations at finite temperature.

All discrete summations of physical quantities are changed to integrations taking care of spin-isospin degrees of freedom [32]. In order to compute thermodynamic functions, one needs to solve self-consistent equations for $M^{*}$; then, $\rho_{B}, \omega_{\mu}$ and other quantities are determined. In finite temperature calculations, main contributions from numerical integrations are generated in the range, $0 \leq k \lesssim 2 k_{F}(T)$; numerical convergences are checked carefully. The baryon density is expressed as (19) which is the function of $\left(n_{\kappa_{i}}-\bar{n}_{\kappa_{i}}\right)$, and because of the emergence of anti-particles, the ground state of Fermi-sphere is slow to increase at finite temperatures.

In Figure 3, effective masses are shown for $T=10, T=30$ and $T=50 \mathrm{MeV}$. The temperature effect is small on effective masses, and the result of $T=10 \mathrm{MeV}$ is almost similar to the one at $T=0$. However, the binding energy curves in Figure 4 indicate that the single particle energy at saturation density is relatively increased with temperature in the range $10 \lesssim T \lesssim 50 \mathrm{MeV}$. The gas-liquid type phase transition is generally expected about saturation density [7] [32], and the binding energy at saturation is enhanced up to a higher density by finite temperature effects (see, Figure 4 at $T=30$ and $T=50$ ). The effect of enhancement at saturation density continues to affect calculations of Fermi liquid properties, such as incompressibility, symmetry energy, first sound velocity and so forth.

\section{Landau Parameters at Finite Temperature}

Landau's theory of Fermi liquid is constructed so that quantum statistical properties, such as energy density, pressure, single particle energy and self-energies are consistent with macroscopic conservation laws, in other words, thermodynamic relations [4] [24]-[28] [34]-[36]. The requirement is not trivially true in an approximation, which is self-consistently related to Hamiltonian or Lagrangian formalism and approximation methods. The validity and applicability of Fermi-liquid theory are confined in the Fermi energy range, finite temperature and density which reasonably maintain $E\left(k_{F}\right)=\mu_{T}$, or in non-equilibrium systems close to their equilibria. Landau's Fermi-liquid properties are discussed at densities where solutions to the self-consistent single particle energy, (29), exist.

Based on the results in the sec. 3, Landau theory of nuclear matter can be rigorously discussed, since the current nonlinear $(\sigma, \omega)$ approximation maintains macroscopic conservation laws which can be rigorously proved with the fundamental relation, $\delta \mathcal{E} / \delta n_{i}=E\left(k_{F}\right)$, in the current approximation. Landau parameters are calculated from self-consistent single particle energy, $\varepsilon_{i}$, and functional derivative of $\varepsilon_{i}$ with respect to particle distributions, $n_{i}$ or $\bar{n}_{i}$, in the current approximation.

Landau parameters are self-consistently computed at the Fermi surface defined by (29): $E\left(k_{F}(T)\right)=\mu_{T}$, and finite temperature effects on Fermi-liquid properties are compared to calculations at $T=0$. The functional derivative of energy density, $\delta \mathcal{E} / \delta n_{i}$, should be performed as functions of momentum $\kappa$ in the sec. 3 , but because Fermi liquid properties are defined in the rest frame of nuclear matter, physical quantities are evaluated in the limit $j_{B} \rightarrow 0$ (or $\omega_{i} \rightarrow 0$ ) and the rest frame of nuclear matter (or comoving frame $\boldsymbol{v}=0$ ). Hence, after the functional derivative and in the limit $j_{B} \rightarrow 0$ and $\boldsymbol{v}=0$, the variable $\boldsymbol{\kappa}$ can be regarded as $\boldsymbol{k}$ in the evaluation of effective mass $M^{*}$ and $\omega_{0}$ in Equations (20) and (21).

The quasiparticle energies are directly obtained from the functional derivative of the energy density, (22), with respect to quasiparticle distribution function of baryons and anti-baryons, $n(\boldsymbol{\kappa}, E(\boldsymbol{\kappa}))$ and $\bar{n}(\boldsymbol{\kappa}, E(\boldsymbol{\kappa}))$. For simplicity, we denote $\boldsymbol{\kappa}_{i} \rightarrow i, \boldsymbol{\kappa}_{j} \rightarrow j$ in the following calculations. The baryon and anti-baryon quasiparticle energies, $\varepsilon_{i}$ and $\bar{\varepsilon}_{i}$, are obtained:

$$
\begin{gathered}
\varepsilon_{i}=\frac{\delta \mathcal{E}}{\delta n_{i}}=E^{*}\left(\boldsymbol{\kappa}_{i}\right)+\frac{g_{\omega}^{2}}{m_{\omega}^{* 2}} \rho_{B}, \\
\bar{\varepsilon}_{i}=\frac{\delta \mathcal{E}}{\delta \bar{n}_{i}}=E^{*}\left(\boldsymbol{\kappa}_{i}\right)-\frac{g_{\omega}^{2}}{m_{\omega}^{* 2}} \rho_{B} .
\end{gathered}
$$

The functional derivatives of the quasiparticle energy with respect to baryon and antibaryon distribution functions will generate the baryon-baryon $(N N)$, baryon-antibaryon $(N \bar{N})$, and $(\bar{N} \bar{N})$ interactions: 


$$
\left(\begin{array}{ll}
f_{i j} & f_{i \bar{j}} \\
f_{\overline{i j}} & f_{\overline{i j}}
\end{array}\right)=\left(\begin{array}{ll}
\delta \varepsilon_{i} / \delta n_{j} & \delta \varepsilon_{i} / \delta \bar{n}_{j} \\
\delta \bar{\varepsilon}_{i} / \delta n_{j} & \delta \bar{\varepsilon}_{i} / \delta \bar{n}_{j}
\end{array}\right),
$$

where

$$
\begin{aligned}
& f_{i j}=\frac{\delta \varepsilon_{i}}{\delta n_{j}}=\frac{\boldsymbol{\kappa}_{i} \cdot \frac{\delta \boldsymbol{\kappa}_{i}}{\delta n_{j}}+M^{*} \frac{\delta M^{*}}{\delta n_{j}}}{E^{*}\left(\boldsymbol{\kappa}_{i}\right)}+\frac{g_{\omega}^{2}}{m_{\omega}^{* 2}}\left(1-\frac{\rho_{B}}{m_{\omega}^{*}} \frac{\delta m_{\omega}^{*}}{\delta n_{j}}\right), \\
& f_{i \bar{j}}=\frac{\delta \varepsilon_{i}}{\delta \bar{n}_{j}}=\frac{\boldsymbol{\kappa}_{i} \cdot \frac{\delta \boldsymbol{\kappa}_{i}}{\delta \bar{n}_{j}}+M^{*} \frac{\delta M^{*}}{\delta \bar{n}_{j}}}{E^{*}\left(\boldsymbol{\kappa}_{i}\right)}-\frac{g_{\omega}^{2}}{m_{\omega}^{* 2}}\left(1+\frac{\rho_{B}}{m_{\omega}^{*}} \frac{\delta m_{\omega}^{*}}{\delta \bar{n}_{j}}\right) . \\
& f_{\overline{i j}}=\frac{\delta \bar{\varepsilon}_{i}}{\delta n_{j}}=\frac{\boldsymbol{\kappa}_{i} \cdot \frac{\delta \boldsymbol{\kappa}_{i}}{\delta n_{j}}+M^{*} \frac{\delta M^{*}}{\delta n_{j}}}{E^{*}\left(\boldsymbol{\kappa}_{i}\right)}-\frac{g_{\omega}^{2}}{m_{\omega}^{* 2}}\left(1-\frac{\rho_{B}}{m_{\omega}^{*}} \frac{\delta m_{\omega}^{*}}{\delta n_{j}}\right), \\
& f_{\overline{i j}}=\frac{\delta \bar{\varepsilon}_{i}}{\delta \bar{n}_{j}}=\frac{\boldsymbol{\kappa}_{i} \cdot \frac{\delta \boldsymbol{\kappa}_{i}}{\delta \bar{n}_{j}}+M^{*} \frac{\delta M^{*}}{\delta \bar{n}_{j}}}{E^{*}\left(\boldsymbol{\kappa}_{i}\right)}+\frac{g_{\omega}^{2}}{m_{\omega}^{* 2}}\left(1+\frac{\rho_{B}}{m_{\omega}^{*}} \frac{\delta m_{\omega}^{*}}{\delta \bar{n}_{j}}\right) .
\end{aligned}
$$

The closed forms of the coupled equations for the derivatives, $\delta \boldsymbol{\kappa}_{i} / \delta n_{j}, \delta M^{*} / \delta n_{j}, \delta \boldsymbol{\kappa}_{i} / \delta \bar{n}_{j}, \delta M^{*} / \delta \bar{n}_{j}$ and $\delta m_{\omega}^{*} / \delta n_{j}, \delta m_{\omega}^{*} / \delta \bar{n}_{j}$, are obtained from Equations (16), (18) for $\boldsymbol{\kappa}_{i}$, and (10), (20), (21) for $M^{*}$ and $m_{\omega}^{*}$. Noticing that the system will become symmetric with respect to the momentum as $\boldsymbol{\kappa}_{i} \rightarrow \boldsymbol{k}_{i}$ in the limit $\boldsymbol{j}_{B} \rightarrow 0$, we have

$$
\left.\frac{\delta m_{\omega}^{*}}{\delta n_{j}}\right|_{j_{B} \rightarrow 0}=\frac{g_{\omega} g_{\omega 4} \omega_{0}}{6 m_{\omega}^{* 3}} \frac{\delta \rho_{B}}{\delta n_{j}}\left[1+\frac{g_{\omega} g_{\omega 4} \omega_{0} \rho_{B}}{3 m_{\omega}^{* 4}}\right]^{-1},
$$

where $\frac{\delta \rho_{B}}{\delta n_{j}}=1$, and in case of $\bar{n}$ functional derivative, $\frac{\delta \rho_{B}}{\delta \bar{n}_{j}}=-1$.

The functional derivatives of $\boldsymbol{\kappa}$ and $M^{*}$ produce the following closed expressions:

$$
\begin{aligned}
& \left.\frac{\delta \kappa}{\delta n_{j}}\right|_{j_{B} \rightarrow 0}=-\frac{g_{\omega}^{2}}{m_{\omega}^{2}} \frac{\boldsymbol{k}_{j}}{E^{*}\left(k_{j}\right)}\left[1+\frac{g_{\omega 4}}{6 m_{\omega}^{2}} \omega_{0}^{2}+\frac{g_{\omega}^{2}}{m_{\omega}^{2}} \sum_{i} \frac{M^{* 2}+\frac{2}{3} k_{i}^{2}}{E_{\left(k_{i}\right)}^{* 3}}\left(n_{i}+\bar{n}_{i}\right)\right]^{-1}, \\
& \left.\frac{\delta M^{*}}{\delta n_{j}}\right|_{j_{B} \rightarrow 0}=-\frac{g_{\sigma}^{2}}{m_{\sigma}^{2}} \frac{M^{*}}{E^{*}\left(k_{j}\right)}\left[1+\frac{g_{\sigma 3}}{m_{\sigma}^{2}} \phi+\frac{g_{\sigma 4}}{2 m_{\sigma}^{2}} \phi^{2}+\frac{g_{\sigma}^{2}}{m_{\sigma}^{2}} \sum_{i} \frac{k_{i}^{2}}{E_{\left(k_{i}\right)}^{* 3}}\left(n_{i}+\bar{n}_{i}\right)\right]^{-1} .
\end{aligned}
$$

Since $\boldsymbol{j}_{B}$ and $M^{*}$ are symmetric with respect to the baryon and antibaryon distribution functions, the functional derivatives, $\delta \boldsymbol{\kappa} / \delta n_{i}$ and $\delta \boldsymbol{\kappa} / \delta \bar{n}_{i}, \delta M^{*} / \delta n_{i}$ and $\delta M^{*} / \delta \bar{n}_{i}$ give the same expressions, resulting in $f_{i j}=f_{j i}$, $f_{i j}=f_{\overline{i j}}$ and $f_{\overline{i j}}=f_{\overline{i j}}$.

According to Landau's theory of Fermi liquid [27] [28], Landau parameters are defined by Legendre expansions of $f$ 's with respect to $k_{i}, k_{j}$, taken on the Fermi surface $k_{F}$, and they are expressed as

$$
\left.\left(f_{l}\right)_{i j} \equiv(2 l+1) \int \frac{\mathrm{d} \Omega}{4 \pi} P_{l}\left(\cos \theta_{i j}\right) f_{i j}\right|_{\left|k_{i}\right|=\left|k_{j}\right|=k_{F}},
$$

where $\cos \theta_{i j}=\frac{\boldsymbol{k}_{i} \cdot \boldsymbol{k}_{j}}{\left|k_{F}\right|^{2}}$. Employing the results (32)-(34), we obtain 


$$
\begin{aligned}
& \left(f_{0}\right)_{i j}=-\frac{g_{\sigma}^{2}}{m_{\sigma}^{2}}\left(\frac{M^{*}}{E^{*}\left(k_{F}\right)}\right)^{2}\left[1+\frac{g_{\sigma 3}}{m_{\sigma}^{2}} \phi+\frac{g_{\sigma 4}}{2 m_{\sigma}^{2}} \phi^{2}+\frac{g_{\sigma}^{2}}{m_{\sigma}^{2}} \sum_{i}\left(n_{i}+\bar{n}_{i}\right) \frac{k_{i}^{2}}{E^{*}\left(k_{i}\right)^{3}}\right]^{-1}+\frac{g_{\omega}^{2}}{m_{\omega}^{* 2}}\left(1-\frac{\rho_{B}}{m_{\omega}^{*}} \frac{\delta m_{\omega}^{*}}{\delta n_{j}}\right), \\
& \left(f_{1}\right)_{i j}=-\frac{g_{\omega}^{2}}{m_{\omega}^{2}}\left(\frac{k_{F}}{E^{*}\left(k_{F}\right)}\right)^{2}\left[1+\frac{g_{\omega 4}}{6 m_{\omega}^{2}} \omega_{0}^{2}+\frac{g_{\omega}^{2}}{m_{\omega}^{2}} \sum_{i}\left(n_{i}+\bar{n}_{i}\right) \frac{M^{* 2}+\frac{2}{3} k_{i}^{2}}{E^{*}\left(k_{i}\right)^{3}}\right]^{-1},
\end{aligned}
$$

and $f_{l}=0$ for $l \geq 2$. The Landau parameter $f_{0}$ contains symmetric and asymmetric contributions for $n(k)$ and $\bar{n}(k)$, but $f_{1}$ is symmetric for $n(k)$ and $\bar{n}(k)$.

The relativistic density of states at the Fermi surface is defined by the use of $\delta$-function as $N_{F}(T)=\sum_{i} \delta\left(\varepsilon_{i}-\mu_{T}\right)$, and it is obtained in Hartree approximation [34]-[36],

$$
N_{F}(T)=\sum_{i} \delta\left(\varepsilon_{i}-\mu_{T}\right)=\frac{\zeta k_{F} E^{*}\left(k_{F}\right)}{2 \pi^{2}},
$$

where $\zeta$ is the spin-isospin degeneracy factor: $\zeta=2$ for neutron matter, $\zeta=4$ for nuclear matter. The dimensionless Landau parameters are defined by

$$
\begin{aligned}
& \left(F_{0}\right)_{i j}=N_{F}(T)\left(f_{0}\right)_{i j}, \\
& \left(F_{0}\right)_{i j}=N_{F}(T)\left(f_{0}\right)_{i j}, \\
& \left(F_{1}\right)_{i j}=N_{F}(T)\left(f_{1}\right)_{i j},
\end{aligned}
$$

and the parameters, $\left(F_{0}\right)_{i j}$ and $\left(F_{1}\right)_{i j}$ against the baryon density $\rho_{B}(T)$, are shown in Figure 5, and $\left(F_{0}\right)_{i j}$ in Figure 6.

In the nonlinear $(\sigma, \omega)$ calculation, the values of Landau parameters, $F_{0}$ and $F_{1}$, become smaller compared to the linear $(\sigma, \omega)$ calculation (LHA). In addition, the finite temperature contribution shifts the density dependence of Landau parameters to a high density, resulting in the decrease of the magnitude of Landau parameters. The Figure 6 shows that baryon-antibaryon, $(N \bar{N})$, interactions contribute negative in all densities, and it indicates that $(N \bar{N})$ many-particle system is unstable. The many-body systems of $\left(F_{0}\right)_{i j}$ and $\left(F_{0}\right)_{\overline{i j}}$ (if it exists) are stable.

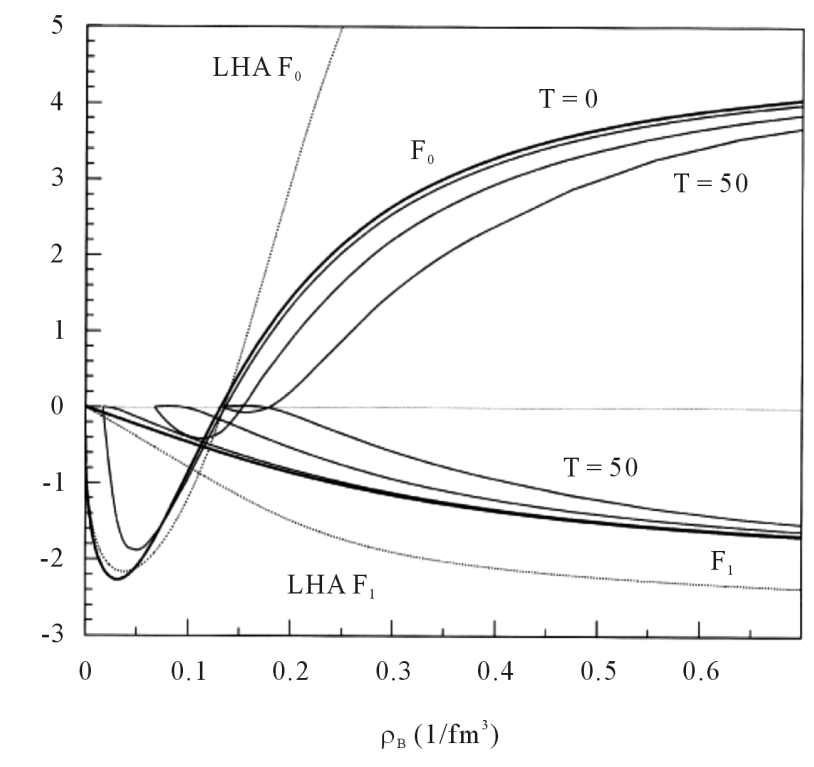

Figure 5. Landau parameters $F_{0}$ and $F_{1}$; LHA (dotted-line at $T=0)$ and NHA (solid-lines at $T=0,10,30,50 \mathrm{MeV}$ ). 


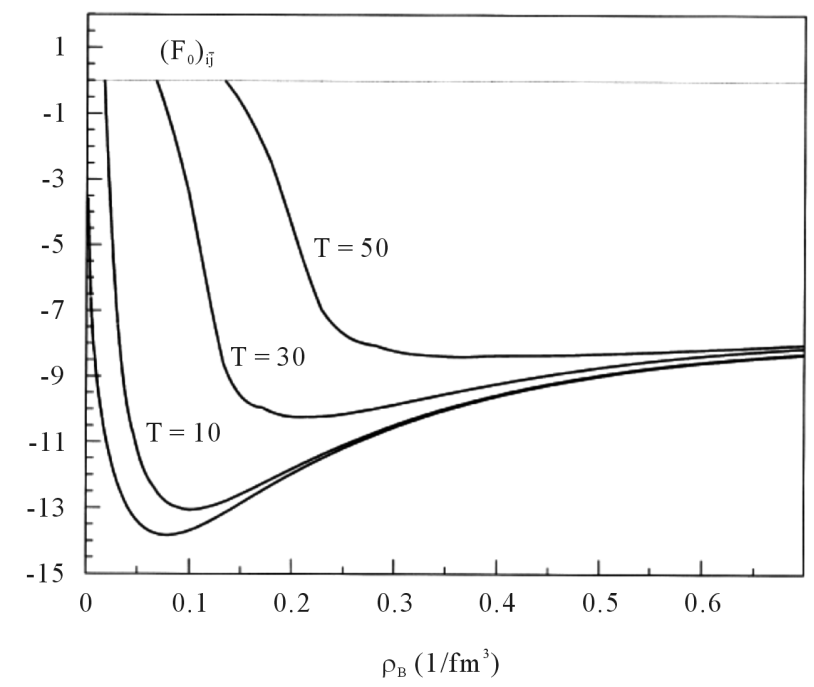

Figure 6. $N \bar{N}$ Landau parameters, $\left(F_{0}\right)_{i \bar{j}}$, which give negative contributions in all densities.

In the current finite temperature Fermi liquid calculation, there are only two components corresponding to baryon-baryon $(N N)$ and baryon-antibaryon $(N \bar{N})$ interactions (32), and we introduce

$$
\begin{aligned}
& \left(f_{l}\right)_{i j}=\left(f_{l}\right)_{i j}^{s}+\left(f_{l}\right)_{i j}^{a} \\
& \left(f_{l}\right)_{i \bar{j}}=\left(f_{l}\right)_{\overline{i j}}=\left(f_{l}\right)_{i j}^{s}-\left(f_{l}\right)_{i j}^{a},
\end{aligned}
$$

where $\left(f_{l}\right)_{i j}^{s}$ and $\left(f_{l}\right)_{i j}^{a}$ are baryon symmetric and baryon antisymmetric parts of the quasiparticle interactions. From Equation (32), one can obtain

$$
\begin{aligned}
& \left(f_{0}\right)_{i j}^{s}=-\frac{g_{\sigma}^{2}}{m_{\sigma}^{2}}\left(\frac{M^{*}}{E^{*}\left(k_{F}\right)}\right)^{2}\left[1+\frac{g_{\sigma 3}}{m_{\sigma}^{2}} \phi+\frac{g_{\sigma 4}}{2 m_{\sigma}^{2}} \phi^{2}+\frac{g_{\sigma}^{2}}{m_{\sigma}^{2}} \sum_{i}\left(n_{i}+\bar{n}_{i}\right) \frac{k_{i}^{2}}{E^{*}\left(k_{i}\right)^{3}}\right]^{-1}, \\
& \left(f_{0}\right)_{i j}^{a}=\frac{g_{\omega}^{2}}{m_{\omega}^{* 2}}\left\{1-\frac{\rho_{B}}{2 m_{\omega}^{*}}\left(\frac{\delta m_{\omega}^{*}}{\delta n_{j}}-\frac{\delta m_{\omega}^{*}}{\delta \bar{n}_{j}}\right)\right\}=\frac{g_{\omega}^{2}}{m_{\omega}^{* 2}}\left\{1-\frac{g_{\omega} g_{\omega 4} \omega_{0} \rho_{B}}{6 m_{\omega}^{* 4}}\left(1+\frac{g_{\omega} g_{\omega 4} \omega_{0} \rho_{B}}{3 m_{\omega}^{* 4}}\right)^{-1}\right\}, \\
& \left(f_{1}\right)_{i j}^{s}=-\frac{g_{\omega}^{2}}{m_{\omega}^{2}}\left(\frac{k_{F}}{E^{*}\left(k_{F}\right)}\right)^{2}\left[1+\frac{g_{\omega 4}}{6 m_{\omega}^{2}} \omega_{0}^{2}+\frac{g_{\omega}^{2}}{m_{\omega}^{2}} \sum_{i}\left(n_{i}+\bar{n}_{i}\right) \frac{M^{* 2}+\frac{2}{3} k_{i}^{2}}{E^{*}\left(k_{i}\right)^{3}}\right]^{-1}, \\
& \left(f_{1}\right)_{i j}^{a}=0 .
\end{aligned}
$$

They are expanded in a series of Legendre polynomials as

$$
\left(f_{l}\right)_{i j}^{s, a}=\sum_{l=0}^{\infty} f_{l}^{s, a} P_{l}\left(\cos \theta_{i j}\right),
$$

and the dimensionless symmetric and antisymmetric Landau parameters, $F_{l}^{s}$ and $F_{l}^{a}$, are defined by

$$
F_{l}^{s}=N_{F}(T) f_{l}^{s}, F_{l}^{a}=N_{F}(T) f_{l}^{a} .
$$

It is easy to see $F_{0}^{s}+F_{0}^{a}=\left(F_{0}\right)_{i j}$, and $F_{1}^{s}=\left(F_{1}\right)_{i j}, F_{l}^{a}=0$ for $l \geq 2$. The parameters $\left(F_{0}^{s}\right)_{i j}$ and $\left(F_{0}^{a}\right)_{i j}$ are shown in Figure 7, and Fermi surface shifts to a higher density when $T$ is increased. The $(N \bar{N})$ Landau parameter $\left(F_{0}\right)_{i \bar{j}}$ is negative at all densities as shown in Figure 7, because $\left(F_{0}^{s}\right)_{\bar{i} \bar{j}}$ is negative and $\left(F_{0}^{a}\right)_{\overline{i j}}=-\left(F_{0}^{a}\right)_{i j}$. 


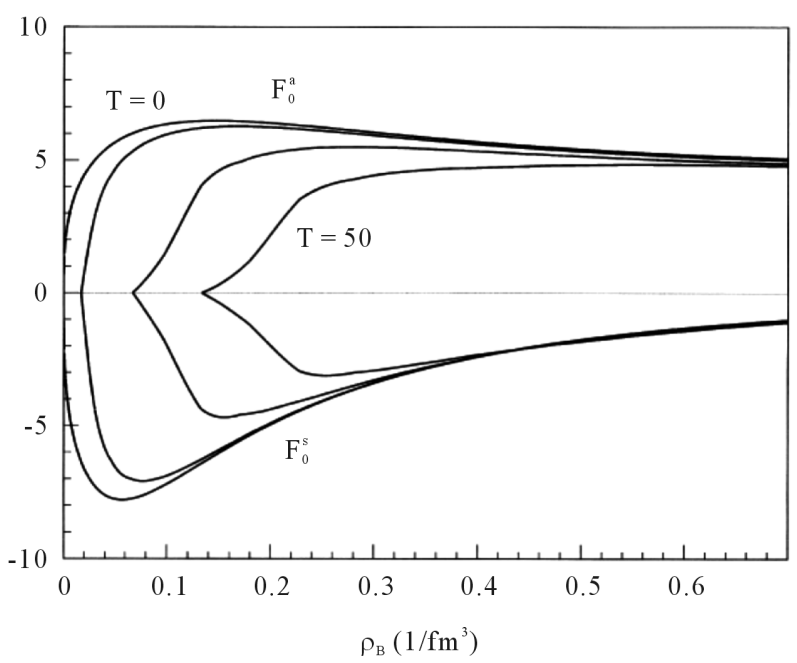

Figure 7. Symmetric and asymmetric Landau parameters, $F_{0}^{s}$ and $F_{0}^{a}$. The onset of parameters is shifted to higher densities according to binding energies in Figure 4.

The attractive interaction coming from the exchange of scalar mesons exceeds the repulsive force due to the exchange of vector mesons at a low density; and the cancellation of large value of scalar and vector meson contributions can be observed in Figure 7 as $F_{0}=F_{0}^{s}+F_{0}^{a}$.

\section{Macroscopic Properties and Scattering of Quasiparticles}

Fermi-liquid properties, such as incompressibility, first sound velocity, symmetry energy and Landau parameters are discussed in a relativistic formalism [8] [45] [46]. The incompressibility, $K$, and symmetry energy, $a_{4}$, are defined by $(T=0)$ :

$$
K=9 \rho_{B} \frac{\partial^{2} E}{\partial \rho_{B}^{2}}, \quad a_{4}=\frac{1}{2} \rho_{B}\left[\left(\frac{\partial^{2} E}{\partial \rho_{3}^{2}}\right)_{\rho_{B}}\right]_{\rho_{3}=0},
$$

where the isovector, $\rho_{3}$, is given by $\rho_{3}=\rho_{p}-\rho_{n}=\left(k_{F_{p}}^{3}-k_{F_{n}}^{3}\right) / 3 \pi^{2}$ using proton and neutron densities.

The first derivative of $\mathcal{E}$ is equal to the single particle energy: $E\left(k_{F}\right)=\partial \mathcal{E} / \partial \rho_{B}$. The incompressibility and symmetry energy given by Landau parameters are,

$$
K=9 \rho_{B}\left(1+F_{0}\right) / N_{F}, \quad a_{4}=\frac{1}{2} \frac{\rho_{B}}{N_{F}}\left(1+F_{0}^{\prime}\right),
$$

where the density of states, $N_{F}$, baryon density, $\rho_{B}$, and Landau parameters are given in Sec. 3. In the current nonlinear $(\sigma, \omega)$ mean-field approximation, symmetry energy can be generally calculated as $a_{4}=\frac{1}{6} \frac{k_{F}^{2}}{E^{*}\left(k_{F}\right)}$ which gives rather small value for $a_{4}$. The Landau parameter, $F_{0}^{\prime}$, is the dimensionless parameter for isovector quasiparticle interactions, and one can directly check $F_{0}^{\prime}=0$ in $(\sigma, \omega)$ model using (43). Serot and Walecka extended $(\sigma, \omega)$ model by including $\rho$-meson, and the model is used to calculate the symmetry energy and isovector Landau parameter [32] [34] [43]-[46], resulting in a reasonable value of $a_{4}$.

The incompressibilities, $K$, and symmetry energies, $a_{4}$, for symmetric nuclear matter in the nonlinear $(\sigma, \omega)$ approximation at $T=0, T=30, T=50$ are shown in Figure 8 and Figure 9 and compared to the result of the linear $(\sigma, \omega)$ mean-field approximation (LHA). The hydrodynamic first sound velocity $C_{1}$ in the relativistic case is given by

$$
C_{1}^{2}=\left(\frac{\partial p}{\partial \mathcal{E}}\right)=\frac{K}{9 \mu}
$$




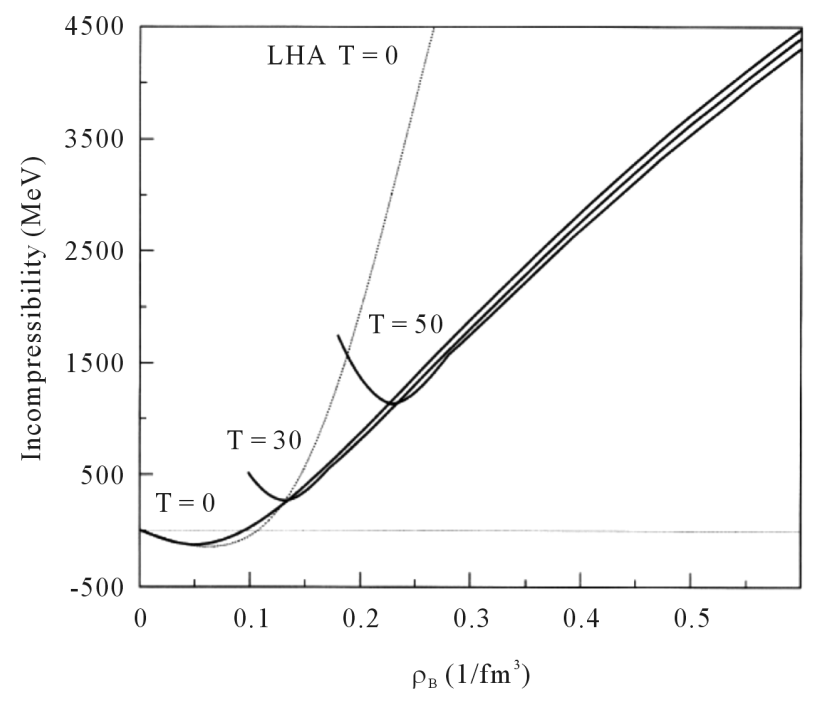

Figure 8. Incompressibilities of symmetric nuclear matter; LHA (dotted-line at $T=0$ ) and NHA (solid-lines at $T=0,30$, $50 \mathrm{MeV}$. The Fermi ground states at low densities are shifted to a higher density according to binding energies in Figure 4.

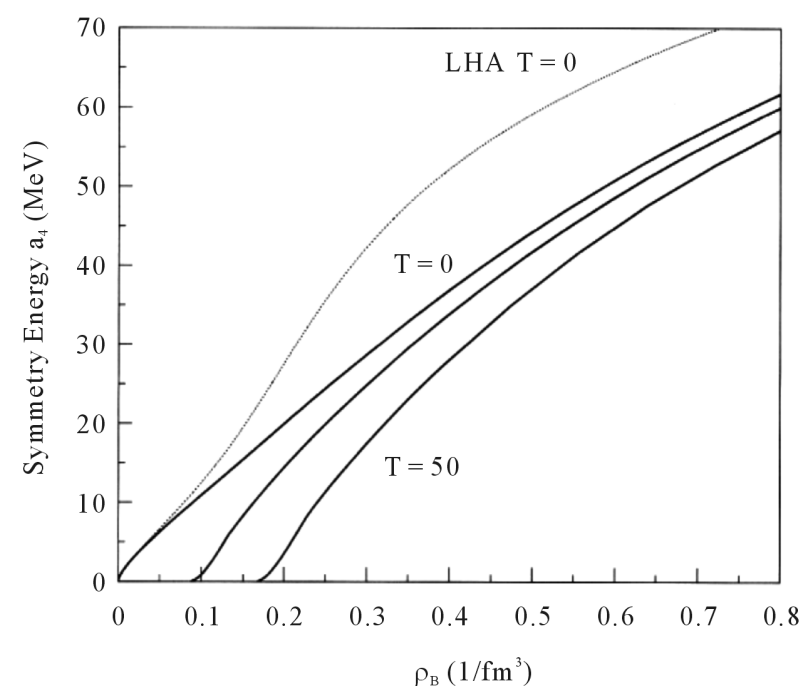

Figure 9. Symmetry energies; LHA at $T=0$ (dotted-line) and NHA (solid-lines at $T=0,30,50 \mathrm{MeV}$ ).

where $p, \mathcal{E}$ are pressure and energy density; $\mu=E\left(k_{F}\right)$ is the chemical potential (single particle energy at the Fermi surface) of the system. The first sound velocities are shown in Figure 10. The average nucleon velocity in the medium is about $0.21 c$ ( $c$ is the velocity of light) at normal nuclear density $(T=0)$, and $C_{1}$ is smaller than the velocity of light at all densities, which is consistent with causality. The effect of temperature to Fermiliquid properties are not large at high densities, however, one should note that the density regions of the gas-liquid phase transition at low densities are slightly extended to higher densities.

The Fermi-liquid properties are significantly reduced at high densities by nonlinear interactions. Because saturation curves are shifted to a higher density and energy (Figure 4), many-body properties such as incompressibility, symmetry energy and first sound velocity at saturation density are accordingly shifted to higher densities (Figure 8, Figure 9 and Figure 10), and it shows that the gas phase or gas-liquid type phase transition is also shifted to higher densities. These characteristic density-dependent properties at finite temperature should be checked experimentally. 


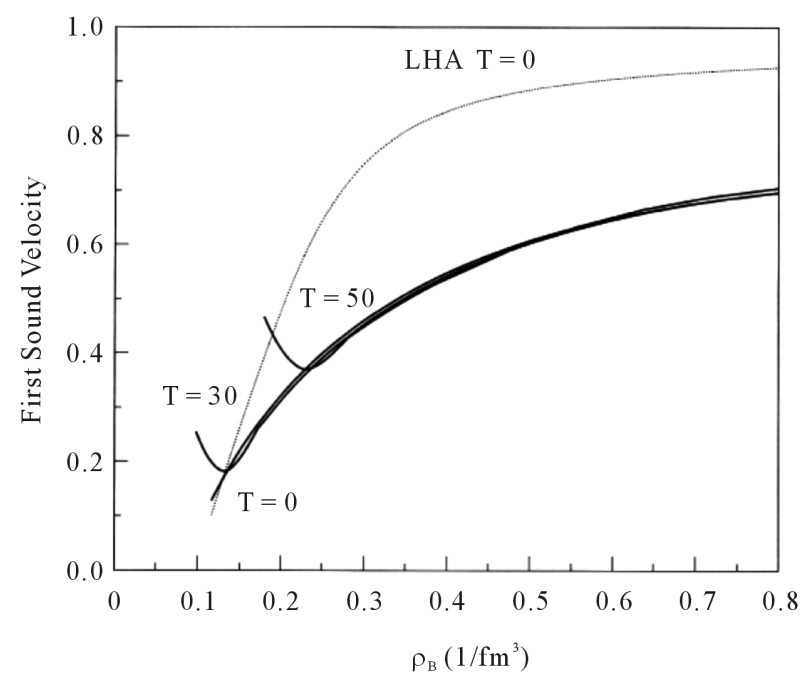

Figure 10. First sound velocities; LHA (dotted-line) and NHA (solid-lines).

The quasiparticle scattering amplitude in the transport theory [26] [27] can be expressed in the current $(\sigma, \omega)$ nonlinear mean-field approximation. The transport equation for quasiparticles yields the equation for the scattering amplitude $A_{p p^{\prime}}\left(q, \varepsilon_{p p^{\prime}}\right)$, where $A_{p p^{\prime}}\left(q, \varepsilon_{p p^{\prime}}\right)$ represents the scattering amplitude for a process in which two quasiparticles with momenta $p$ and $p^{\prime}$ exchange momentum and energy transfer $q=p-p^{\prime}$ and

$\varepsilon_{p p^{\prime}}=\varepsilon(p)-\varepsilon\left(p^{\prime}\right)$. It is assumed that the scattering is nearly forward, $\varepsilon_{p p^{\prime}}$ is bound to be $\varepsilon_{p p^{\prime}} \lesssim T$, but $q$ is moderately large as $0 \leq q \lesssim v v_{F}$, where $v$ is the chemical potential and $v_{F}=\mathrm{d} \varepsilon\left(k_{F}\right) / \mathrm{d} k_{F}$ is the relativistic Fermi velocity. The equation for the corresponding scattering amplitude is defined by,

$$
A_{i j}(q, \omega) \simeq \boldsymbol{f}_{i j}=f_{i j}+\sum_{l} f_{i l}\left(\partial \rho_{B} / \partial \varepsilon_{l}\right) \boldsymbol{f}_{l j} .
$$

The expansions of Legendre polynomials in Equation (35) yield:

$$
N_{F}(T) \boldsymbol{f}_{i j}=\sum_{l}\left\{\boldsymbol{F}_{l}^{s} \pm \boldsymbol{F}_{l}^{a}\right\} P_{l}\left(\cos \theta_{i j}\right),
$$

where

$$
\boldsymbol{F}_{l}^{s}=\frac{F_{l}^{s}}{1+F_{l}^{s} /(2 l+1)}, \boldsymbol{F}_{l}^{a}=\frac{F_{l}^{a}}{1+F_{l}^{a} /(2 l+1)} .
$$

Properties of symmetric nuclear matter at saturation densities are listed. The saturation of the NHA $(T \geq 50)$ cannot be defined.

The transition probabilities of the quasiparticle collisions of baryon-baryon, baryon-antibaryon scattering, $W_{i j}$ and $W_{\overline{i j}}$, are defined as,

$$
W_{i j}(\theta, \phi)=2 \pi\left|A_{i j}(\theta, \phi)\right|^{2}, W_{\overline{i j}}(\theta, \phi)=2 \pi\left|A_{i \bar{j}}(\theta, \phi)\right|^{2},
$$

where $\phi$ is the angle between the plane containing the momentum vectors of incident quasiparticles and the plane containing the momentum vectors of the scattered quasiparticles [26] [27] (note that $\hbar=c=1$ ). Assuming $A_{i j}$ and $A_{i \bar{j}}$ are independent of angles, the order of magnitude estimate of the two quantities is given by

$$
N_{F}(T) A_{i j} \sim\left(\boldsymbol{F}_{0}^{s}+\boldsymbol{F}_{0}^{a}\right), N_{F}(T) A_{i \bar{j}} \sim\left(\boldsymbol{F}_{0}^{s}-\boldsymbol{F}_{0}^{a}\right),
$$

and the average transition probabilities are computed from the Equation (49) by employing Equation (50). Because of assumptions introduced to derive the equation for the scattering amplitude, the validity of Equation (48) should be confined in a density where constraints, $F_{0}^{s}>-(2 l+1)$ and $F_{0}^{a}>-(2 l+1)$, are maintained. Fermiliquid properties of symmetric nuclear matter and Landau parameters are listed in Table 1 . The finite temperature effects are mainly noticeable about saturation and low densities. 
Table 1. Fermi-liquid properties for nuclear matter.

\begin{tabular}{ccccccccccc}
\hline & $M_{N}^{*} / M$ & $m_{\sigma}^{*} / m_{\sigma}$ & $m_{\omega}^{*} / m_{\omega}$ & $K$ & $a_{4}$ & $C_{1}$ & $F_{0}$ & $F_{0}^{s}$ & $F_{0}^{a}$ & $F_{1}^{s}$ \\
\hline LHA $(T=0)$ & 0.54 & 1 & 1 & 541 & 19.3 & 0.26 & 0.56 & -8.62 & 9.18 & -1.15 \\
NHA $(T=0)$ & 0.72 & 1.08 & 1.02 & 333 & 15.3 & 0.22 & 0.41 & -6.07 & 6.48 & -0.66 \\
NHA $(T=10)$ & 0.72 & 1.08 & 1.02 & 356 & 14.5 & 0.21 & 0.27 & -5.98 & 6.25 & -0.62 \\
NHA $(T=30)$ & 0.73 & 1.08 & 1.02 & 362 & 7.94 & 0.21 & -0.08 & -4.57 & 4.49 & -0.26 \\
\hline
\end{tabular}

\section{Concluding Remarks}

The nonlinear $(\sigma, \omega)$ mean-field approximation is extended to finite temperature and applied to properties of nuclear matter by way of Landau's Fermi-liquid theory. The finite temperature mean-field approximations in QHD are thermodynamically consistent relativistic approximations, and Landau's assumption in the theory of Fermi liquid is maintained rigorously. Hence, nonlinear interactions of hadrons and finite temperature effects can be consistently examined in the mean-filed approximation of QHD.

The nonlinear interactions appear as density-dependent and energy-dependent interactions, which manifestly contribute at high densities. On the contrary, finite temperature effects appear at low densities of the Fermi-liquid ground state of nuclear matter and contribute to observables at saturation density. Although finite temperature effects on hadron effective masses are not large, the single particle energy $E(k)$ and Landau parameters, $F_{0}, F_{1}$, are subject to temperature contributions at low densities. The finite temperature effects of symmetry energy, incompressibility and sound velocity should be reexamined by including $\rho$-meosn [43]-[46].

At finite temperature, the Fermi surface is smeared out, which is observed by comparing Fermi momentums, $k_{F}(0)$ at $T=0$ and $k_{F}(T)$. The Fermi momentums are $k_{F}(T)<k_{F}(0)$ at low densities, and they are almost equal, $k_{F}(T) \sim k_{F}(0)$, at high densities, restoring a sharp Fermi surface. When $T \gtrsim 50 \mathrm{MeV}$, the Fermi surface at saturation density disappears completely. Though effective masses of nucleons and mesons change slightly, the single particle energy, symmetry energy and modifications to Landau parameters should be examined carefully at finite temperature and low densities.

The Landau parameters in scattering amplitude, (48)-(50), should be checked in heavy-ion collision experiments, for instance, whether the modification to Fermi liquid properties or the reduction to Landau parameters is significant or not. Nucleon and meson effective masses depend on nonlinear and density interactions, which become noticeable above saturation densities. Hence, nonlinear and density modifications to physical quantities are important from saturation to higher densities, whereas finite temperature modifications would be important from low to saturation densities. From the current Hartree approximation, it is suggested that Fermi-liquid properties are fairly sensitive to variations of temperature at low densities.

Physical quantities sensitive to Landau parameters, $F_{0}^{s}$ and $F_{0}^{a}$, or the density of states at the Fermi surface $N_{F}(T)$ should be checked at low density and finite temperature. The specific heat depends on the density of states and linearly on temperature in low temperature limit [26]. Properties of Fermi liquid would be moderately stable at high densities and temperatures. There may be certain static properties of nucleus sensitive to low density and temperature, charge and electromagnetic interactions [24]. Physical quantities directly related to the density of states at the Fermi surface should be examined at a low temperature and a low density nuclear system. In addition, non-equilibrium process should be included. Fermi liquid properties at finite temperature with nonequilibrium effects may exhibit different results from those obtained by approximations at $T=0$.

The exchange interaction in the Dirac-Hartree-Fock approximation in QHD is applied to obtain the value of Landau parameters [35] [36]. The similar analyses may be carried out in more sophisticated approximations in QHD, such as the effective chiral $(\sigma, \pi, \omega)$ mean-field [8] [31] approximation, the chiral $(\sigma, \pi, \omega)$ Hartee-Fock approximation [47], and the chiral $(\sigma, \pi, \omega, \rho)$ model [48]. These analyses are necessary for applications to neutron stars as high density matter [6]-[8], nuclear fission and cluster radioactivity [40]-[42]. It is important for the theory of finite temperature quantum liquids to examine heavy-ion collision experiments at a low temperature and a density, so as to clarify the validity and applicability of quasiparticle approach in nuclear matter.

\section{References}

[1] Walecka, J.D. (1974) A Theory of Highly Condensed Matter. Annals of Physics, 83, 491-529. 
http://dx.doi.org/10.1016/0003-4916(74)90208-5

[2] Haar, B. and Malfliet, R. (1987) Nucleons, Mesons and Deltas in Nuclear Matter a Relativistic Dirac-Brueckner Approach. Physics Reports, 149, 207-286. http://dx.doi.org/10.1016/0370-1573(87)90085-8

[3] Holinde, K. (1981) Two-Nucleon Forces and Nuclear Matter. Physics Reports, 68, 121-188. http://dx.doi.org/10.1016/0370-1573(81)90188-5

[4] Fetter, A.L. and Walecka, J.D. (2003) Quantum Theory of Many-Particle Systems. Dover Pub, New York.

[5] Walecka, J.D. (1995) Theoretical Nuclear and Subnuclear Physics. Oxford University Press, New York.

[6] Uechi, S.T. and Uechi, H. (2009) The Density-Dependent Correlations among Observables in Nuclear Matter and Hyperon-Rich Neutron Stars. Advances in High Energy, 2009, Article ID: 640919.

[7] Serot, B.D. and Uechi, H. (1987) Neutron Stars in Relativistic Hadron-Quark Models. Annals of Physics, 179, $272-293$. http://dx.doi.org/10.1016/0003-4916(87)90137-0

[8] Uechi, H., Uechi, S.T. and Serot, B.D. (2012) Neutron Stars: The Aspect of High Density Matter, Equations of State and Related Observables. Nova Science Pub, Hauppauge.

[9] Jacob, M. and Thanh Van, J.T. (1982) Quark Matter Formation and Heavy Ion Collisions: A General Review and Status. Physics Reports, 88, 321-413. http://dx.doi.org/10.1016/0370-1573(82)90083-7

[10] Uechi, S.T. and Uechi, H. (2015) Hardon-Quark Hybrid Stars Constructed by the Nonlinear $\sigma-\omega$ - $\rho$ Mean-Field Model and MIT-Bag Model. Open Access Library Journal, 2, e2012. http://dx.doi.org/10.4236/oalib.1102012

[11] Lourenco, O., Dura, M., Delfino, A. and Malheiro, M. (2011) Hadron-Quark Phase Transition in a Hadronic and Polyakov-Nambu-Jona Lasinio Models Perspective. Physical Review D, 84, Article ID: 125034. http://dx.doi.org/10.1103/PhysRevD.84.125034

[12] Weldon, H.A. (1982) Covariant Calculations at Finite Temperature: The Relativistic Plasma. Physical Review D, 26, 1394. http://dx.doi.org/10.1103/PhysRevD.26.1394

[13] Weldon, H.A. (1983) Simple Rules for Discontinuities in Finite-Temperature Field Theory. Physical Review D, 28, 2007.

[14] Landsman, N.P. and Van Weert, Ch.G. (1987) Real- and Imaginary-Time Field Theory at Finite Temperature and Density. Physics Reports, 145, 141-249. http://dx.doi.org/10.1016/0370-1573(87)90121-9

[15] Furnstahl, R.J. and Serot, B.D. (1990) Covariant Mean-Field Calculations of Finite-Temperature Nuclear Matter. Physical Review C, 41, 262. http://dx.doi.org/10.1103/PhysRevC.41.262

[16] Furnstahl, R.J. and Serot, B.D. (1991) Covariant Feynman Rules at Finite Temperature: Time-Path Formulation. Physical Review C, 44, 2141-2174. http://dx.doi.org/10.1103/PhysRevC.44.2141

[17] Baym, G. and Kadanoff, L.P. (1961) Conservation Laws and Correlation Functions. Physical Review, 124, $287-299$. http://dx.doi.org/10.1103/PhysRev.124.287

[18] Baym, G. (1962) Self-Consistent Approximations in Many-Body Systems. Physical Review, 127, 1391-1401. http://dx.doi.org/10.1103/PhysRev.127.1391

[19] Day, B.D. (1978) Current State of Nuclear Matter Calculations. Reviews of Modern Physics, 50, 495-521. http://dx.doi.org/10.1103/RevModPhys.50.495

[20] Hugenholtz, N.M. and Van Hove, L. (1958) A Theorem on the Single Particle Energy in a Fermi Gas with Interaction. Physica, 24, 363-376. http://dx.doi.org/10.1016/S0031-8914(58)95281-9

[21] Serot, B.D. (1992) Quantum Hadrodynamics. Reports on Progress in Physics, 55, 1855-1946. http://dx.doi.org/10.1088/0034-4885/55/11/001

[22] Uechi, H. (2001) Self-Consistent Structure in a Relativistic Dirac-Hartree-Fock Approximation. Nuclear Physics A, 696, 511-526. http://dx.doi.org/10.1016/S0375-9474(01)01139-3

[23] Uechi, H. (2004) The Theory of Conserving Approximations and the Density Functional Theory in Approximations for Nuclear Matter. Progress of Theoretical Physics, 111, 525-543. http://dx.doi.org/10.1143/PTP.111.525

[24] Migdal, A.B. (1968) Nuclear Theory: The Quasiparticle Method, W.A. Benjamin, Inc., New York. Migdal, A.B. (1967) Theory of Finite Fermi Systems. John Wiley, New York.

[25] Petkov, I.Z. and Stoitsov, M.V. (1991) Nuclear Density Functional Theory. Oxford University Press, Oxford.

[26] Baym, G. and Pethick, C. (1978) Landau Fermi-Liquid Theory and Low Temperature Properties of Normal Liquid ${ }^{3}$ He. In: Bennemann, K.H. and Ketterson, J.B., Eds., The Physics of Liquid and Solid Helium, Part 2, Wiley, New York, 1-122.

[27] Pines, D. and Nozières, P. (1989) The Theory of Quantum Liquids. Vol. 1, Addison-Wesley, California.

[28] Nozieres, P. (1997) Theory of Interacting Fermi Systems. Perseus Publishing, Cambridge. 
[29] Kohn, W. and Sham, L.J. (1965) Self-Consistent Equations Including Exchange and Correlation Effects. Physical Review, 140, A1133-A1138. http://dx.doi.org/10.1103/physrev.140.a1133

[30] Kohn, W. (1999) Nobel Lecture: Electronic Structure of Matter-Wave Functions and Density Functionals. Reviews of Modern Physics, 71, 1253-1266. http://dx.doi.org/10.1103/RevModPhys.71.1253

[31] Uechi, S.T. and Uechi, H. (2010) Density-Dependent Properties of Hadronic Matter in an Extended Chiral $(\sigma, \pi, \omega)$ Mean-Field Model. Open Access Library Journal, 2, 1-18.

[32] Serot, B.D. and Walecka, J.D. (1986) Advances in Nuclear Physics. Edited by Negele, J.W. and Vogt, E., Vol. 16, Plenum, New York.

[33] Müller, H. and Serot, B.D. (1995) Phase Transitions in Warm, Asymmetric Nuclear Matter. Physical Review C, 52, 2072-2091. http://dx.doi.org/10.1103/PhysRevC.52.2072

[34] Matsui, T. (1981) Fermi-Liquid Properties of Nuclear Matter in a Relativistic Mean-Field Theory. Nuclear Physics A, 370, 365-388. http://dx.doi.org/10.1016/0375-9474(81)90103-2

[35] Uechi, H. (1992) Landau Fermi-Liquid Theory and Approximations in the Quantum Hadrodynamical Model. Nuclear Physics A, 541, 397-412. http://dx.doi.org/10.1016/0375-9474(92)90183-K

[36] Uechi, H. (1990) Constraints on the Self-Consistent Relativistic Fermi-Sea Particle Formalism in the Quantum Hadrodynamical Model. Physical Review C, 41, 744-752. http://dx.doi.org/10.1103/PhysRevC.41.744

[37] Uechi, H. (2008) Density-Dependent Correlations between Properties of Nuclear Matter and Neutron Stars in a Nonlinear $\sigma-\omega-\rho$ Mean-Field Approximation. Nuclear Physics A, 799, 181-209. http://dx.doi.org/10.1016/j.nuclphysa.2007.11.003

[38] Uechi, H. (2008) Correlations between Saturation Properties of Isospin Symmetric and Asymmetric Nuclear Matter in a Nonlinear $\sigma-\omega-\rho$ Mean-Field Approximation. Advanced Studies in Theoretical Physics, 2, 519-548.

[39] Hakim, R. (2011) Introduction to Relativistic Statistical Mechanics, Classical and Quantum. World Scientific Publishing, Singapore.

[40] Hooshyar, M.A., Reichstein, I. and Malik, F.B. (2005) Nuclear Fission and Cluster Radioactivity. Springer-Verlag, Berlin.

[41] Krappe, H.J. and Pomorski, K. (2012) Theroy of Nuclear Fission. Springer-Verlag, Berlin. http://dx.doi.org/10.1007/978-3-642-23515-3

[42] Beck, C. Ed. (2014) Clusters in Nuclei. Volume 3, Springer-Verlag, Berlin.

[43] Serot, B.D. (1979) A Relativistic Nuclear Field Theory with $\pi$ and $\rho$ Mesons. Physics Letters B, 86, 146-150. (Erratum: Physics Letters B, 87, 403.) http://dx.doi.org/10.1016/0370-2693(79)90804-9

[44] Serot, B.D. and Walecka, J.D. (1979) Properties of Finite Nuclei in a Relativistic Quantum Field Theory. Physics Letters B, 87, 172-176. http://dx.doi.org/10.1016/0370-2693(79)90957-2

[45] Uechi, H. (1989) Fermi-Liquid Properties of Nuclear Matter in a Dirac-Hartree-Fock Approximation. Nuclear Physics $A, \mathbf{5 0 1}, 813-834$. http://dx.doi.org/10.1016/0375-9474(89)90162-0

[46] Uechi, H. (2006) Properties of Nuclear and Neutron Matter in a Nonlinear $\sigma-\omega-\rho$ Mean-Field Approximation with Self-and Mixed-Interactions. Nuclear Physics A, 780, 247-273. http://dx.doi.org/10.1016/j.nuclphysa.2006.10.015

[47] Uechi, H. (2012) The Effective Chiral Model of Quantum Hadrodynamics Applied to Nuclear Matter and Neutron Stars. Journal of Applied Mathematics and Physics, 3, 114-123.

[48] Serot, B.D. and Walecka, J.D. (1992) Chiral QHD with Vector Mesons. Acta Physica Polonica B, 23, 655-679. 
Submit or recommend next manuscript to OALib Journal and we will provide best service for you:

- Publication frequency: Monthly

- 9 subject areas of science, technology and medicine

- Fair and rigorous peer-review system

- Fast publication process

- Article promotion in various social networking sites (LinkedIn, Facebook, Twitter, etc.)

- Maximum dissemination of your research work

Submit Your Paper Online: Click Here to Submit

Contact Us: service@oalib.com 